\section{GALAXIES}

\section{New Local Group}

from our Observatories Correspondent IN 1968, Paulo Maffei discovered two faint, diffuse, objects in the Milky Way in the course of a survey at the Asiago Observatory in Italy with film sensitive to the near infrared. Maffei made no suggestion as to the nature of these objects. Now in the January issue of the Astrophysical Journal Letters (163, L25; 1971) a group of nine optical and radio astronomers at the Lick, Leuschner and Hale Observatories in California suggest that the brighter object, Maffei 1 , is in fact a giant elliptical galaxy and moreover that it is possibly a member of the Local Group of galaxies.

Both of Maffei's objects are within half a degree of the galactic plane. As a result, they are heavily reddened and obscured by interstellar dust in our galaxy. The extinction of Maffei 1 in the visible is estimated to be 5 magnitudes (a factor of 100 !) but in the near infrared it is much less. The California astronomers show that at $2.2 \mu \mathrm{m}$ the flux from the central $8^{\prime \prime}$ of Maffei 1 is comparable with that from a similar area in the centre of M31. After a correction for interstellar extinction the overall energy distribution of Maffei 1 is similar to that of the central bulge of M31 which in turn resembles the centres of giant elliptical galaxies. The spectrum of the object contains absorption features; for example, TiO bands and the sodium $D$ lines. These features have the strengths characteristic of the nuclei of giant elliptical or $\mathrm{Sa}$ and $\mathrm{Sb}$ spiral galaxies.

Only crude limits are placed on the distance of Maffei 1. Its radial velocity is only $10 \pm 50 \mathrm{~km} \mathrm{~s}^{-1}$ relative to the Sun or $16.5 \pm 50 \mathrm{~km} \mathrm{~s}^{-1}$ relative to the centre of our galaxy. This velocity is too small to establish Maffei 1 as being necessarily outside the galaxy. Its extragalactic nature is established by two pieces of evidence. First, near infrared photographs show that it has the form typical of an E3 or E4 galaxy; moreover, the radial distribution of the surface brightness is more characteristic of an elliptical galaxy than the central bulge of the spiral. Second, it has the energy distribution characteristic of a massive galaxy.

Because the redshift of Maffei 1 is too low to use the Hubble constant, the Californian group has used dynamical arguments to estimate its distance. The mass-to-light ratio found typically for giant elliptical galaxies is thirty solar units. The internal velocity dispersion of the stars in Maffei 1 was estimated from the widths of absorption lines in its spectrum to be about $200 \mathrm{~km} \mathrm{~s}^{-1}$. The mass-tolight ratio can be estimated from this quantity and from the observed brightness (corrected for interstellar absorption) of the object. The result is found to depend linearly on the distance; a value of 1.2 megaparsecs gives a mass-to-light ratio of thirty. Its mass is then about $2 \times 10^{11}$ Suns, comparable with the mass of the Milky Way.

Maffei 1 is thus about twice as far away as M31 and must be considered as a possible outlying, massive member of the Local Group. Maffei 1 has too much kinetic energy to be bound to the Local Group, however, unless it contains appreciable mass in the form of interstellar gas.

The Californians are continuing observations of Maffei 2. This is only $40^{\prime}$ arc away from Maffei 1 and is implied to be also a highly obscured member of the Local Group of galaxies.

\section{MOON \\ Lunar Quakes}

from our Geomagnetism Correspondent

IN spite of the widespread publicity given to the rock samples returned from the Moon by Apollos 11 and 12, the most significant lunar experiment--the lunar seismic experiment under the supervision of Dr Gary Latham of the LamontDoherty Geological Observatory-continues to operate on the Moon itself. Seismometers were placed on the Moon by the Apollo 12 astronauts on November 19, 1969, as part of the Apollo Lunar Surface Experiments Package and since then have recorded a series of natural and man-made events. During the first seven months of operation, about 160 natural events were detected, of which at least twenty-six were small moonquakes. The rest were probably meteoroid impacts. In addition, seismic recordings were made of two man-made events-the impact on the lunar surface of the Apollo 12 Lunar Module (LM) ascent stage and the third stage of the Apollo 13 Saturn booster (S-IVB).

Latham et al. report (Science, 170, $620 ; 1970)$ that, although the number of natural events was comparatively large, techniques for deriving the times and positions of these disturbances from their seismograms have proved discouragingly elusive. The evidence for the interpretation of some of these events as shallow moonquakes is thus circumstantial rather than analytical, but is none the less valid for that. For example, all of the supposed moonquakes occurred within three days of the Moon's closest approach to Earth during its monthly cycle; and at least one quake was associated with each monthly perigee. This strongly suggests that the release of seismic energy in the outer shell of the Moon is a result of the gravitational interaction between the Moon and the Earth, for the tidal strains thus set up are maximum at perigee. Even so, the moonquakes were small, the largest events having magnitudes between 1 and 2 on the Richter scale. Earthquakes of this magnitude would, of course, be barely perceptible. The low rate of seismic energy release on the Moon thus suggests that plate tectonics, and all that it implies, is not a lunar phenomenon.

But the most important data have come not so much from the natural events but from the two man-made disturbances - partly because the latter impacts were more energetic but chiefly because precise details of the sources were available. For one thing, body wave seismic velocities in the upper few metres of lunar material are very low indeed $-0.1 \mathrm{~km} \mathrm{~s}^{-1}$ for $\mathrm{LM}$. To Latham et al. this is significant because such low compressional velocities were also obtained, by other workers, in terrestrial laboratory experi-

\title{
MIT's Second Look af Crab Pulsar
}

AT the height of the pulsar excitement in 1969 the X-ray astronomy group at MIT rapidly re-scheduled a rocket flight to see if they could pick up X-ray pulsations from the Crab pulsar to match the optical pulsations that had been recorded. They were just beaten by the NRL group, and the MIT flight carried out on April 27 from White Sands Missile Range became a valuable confirmation of the NRL data. Nature published a preliminary analysis of the MIT data a month after the flight (Bradt et al., 222, 728; 1969), and the group's last word is being published in next Monday's Nature Physical Science (229, 40; 1971). Four groups altogether have now observed $X$-rays from the Crab pulsar (NP0532) with energies between 1 and $10 \mathrm{keV}$, but Rappaport et al. at MIT claim the greatest statistical precision with nearly a third of a million counts from the pulsar and the nebula around it.
Nothing in the new analysis contradicts what has already been published, fortunately, but Rappaport, Bradt and Mayer are able to present their conclusions on the parameters of the X-ray pulses more precisely by including all their data instead of only about half of them. They show that only 9 per cent of the 1.5-10 keV emission from the direction of the Crab is pulsed, and there is a substantial amount of emission after the main pulse occupying the valley preceding the interpulse, which is absent in the optical measurements. There are also significant differences in shape between the X-ray and optical interpulses-and in fact the $\mathrm{X}$-ray interpulse contains more energy than the chief X-ray pulse.

To round off the discussion the MIT group give their data on the spectrum of the pulsar at $\mathrm{X}$-ray wavelengths, which one day will have to be fitted into a general theory of how pulsars work. 